\title{
Review on Pharmacological Activities of the Peptides from Scorpion Buthus
}

\section{Martensii Karsch}

\section{Wang $\mathrm{JH}^{1 \#^{*}}$, Zhang $\mathrm{H}^{2 \#}$, Gao SJ ${ }^{1}$ and Wang $\mathrm{JX}^{1}$}

${ }^{1}$ The Experiment Center of Shandong University of Traditional Chinese Medicine, People's Republic of China

${ }^{2}$ School of Pharmacy, Shandong University of Traditional Chinese Medicine, People's Republic of China

${ }^{*}$ Wang JH and Zhang $\mathrm{H}$ contributed equally to this work

${ }^{*}$ Corresponding author: Wang JH, The Experiment Center of Shandong University of Traditional Chinese Medicine, Jinan, People’s Republic of China 250355, E-mail: wangjihuilaoshi@163.com

Citation: Wang JH, Zhang H, Gao SJ, Wang JX (2015) Review on Pharmacological Activities of the Peptides from Scorpion Buthus Martensii Karsch. J Pharm Drug Devel 3(2): 202. doi: 10.15744/2348-9782.2.202

Received Date: March 07, 2015 Accepted Date: April 21, 2015 Published Date: April 23, 2015

\begin{abstract}
In China, the scorpion Buthus martensii Karsch is used as functional food and medicinal materials. The scorpion, scorpion venoms and their extracts are effective in treating a variety of nervous system diseases such as epilepsy, apoplexy pains and facial paralysis. Apart from these therapeutic effects, the peptides purified from scorpions and their venom also showed antitumor activities, antimicrobial effects and protective effects on vascular. In this review, we reported various pharmacological effects of bioactive peptides purified from scorpion Buthus martensii Karsch so that we can use different effects of these bioactive peptides to treat different diseases and provide reference for the study of scorpion Buthus martensii Karsch in future.
\end{abstract}

Keywords: Buthus martensii Karsch; Bioactive peptides; Pharmacological activities

\section{Introduction}

Scorpion Buthus martensii Karsch (BmK) is one of the most ancient animals widely distributed in Mongolia, Korea and China. In China, scorpion BmK has been one of the indispensable and essential materials traditionally used as Chinese medicine for treating the symptoms associated with convulsive Spasm, cardiovascular and cerebrovascular diseases, inflammation, hepatopathy and tumours for 1000 years since the Sung Dynasty [1,2]. To date, there have been more than a hundred peptides isolated and characterized successively from scorpion $\mathrm{BmK}$ and their venoms. Scorpion venoms are rich in diverse bioactive polypeptides which specifically modulate the activity of $\mathrm{Na}^{+}$- channels [3], $\mathrm{K}^{+}$- channels [4,5], $\mathrm{Cl}^{-}$-channels [6] and $\mathrm{Ca}^{2+}$ - channels [7] and exhibit pharmacological activities.

After the in vivo and in vitro studies, some isolated scorpion peptides have been demonstrated to have considerable therapeutic effects such as antimicrobial activity, antitumor, analgesic activity, anti-epilepsy, anti-vascular disease and so on (Table 1). Although the clinical researches about pharmacological effects of isolated peptides and the extracts of scorpion are few, the scorpion BmK as an alternative and complementary medicine has been widely used.

\begin{tabular}{|c|c|c|}
\hline Pharmacological property & Active peptides & References \\
\hline Antimicrobial activity in vitro and in vivo & $\begin{array}{l}\text { BmKn2, BmKb1 } \\
\text { BmKn2-7 } \\
\text { compounds } 1,2\end{array}$ & $\begin{array}{l}\text { Zeng et al. }{ }^{[11]} \\
\text { Cao et al. }{ }^{[12]} \\
\text { Gao et al. }{ }^{[13]}\end{array}$ \\
\hline Inhibiting the glioma cell migration in vitro & $\mathrm{BmK} \mathrm{CT}$ & Fu et al. ${ }^{[17-20]}$ \\
\hline Antitumor against Ehrlich ascites and S-180 fibrosarcoma in vitro & BmK AGAP-SYPU2 & Shao et al. ${ }^{[21]}$ \\
\hline \multirow[t]{6}{*}{ Antinociceptive activity in vivo } & BmK IT2 & Wang et al. ${ }^{[23]}$ \\
\hline & BmK dITAP3 & Guan et al. ${ }^{[24]}$ \\
\hline & AngP1 & Guan et al. ${ }^{[22]}$ \\
\hline & BmK AGP-SYPU1 & $\begin{array}{l}\text { Wang et al. } .^{[25,26]} \\
\text { Deng et al. }{ }^{[27]}\end{array}$ \\
\hline & BmK AGP-SYPU2 & $\begin{array}{l}\text { Zhang et al. }{ }^{[28,29]} \\
\text { Zhao et al. }{ }^{[30]}\end{array}$ \\
\hline & BmK AGAP & $\begin{array}{l}\text { Liu et al. } .^{[31]} \\
\text { Ma et al. }{ }^{[32]} \\
\text { Cui et al. }{ }^{[33]} \\
\text { Mao et al. } .^{[34]}\end{array}$ \\
\hline
\end{tabular}




\begin{tabular}{|c|c|c|}
\hline Pharmacological property & Active peptides & References \\
\hline & BmKAS & Cui et al. ${ }^{[35]}$ \\
\hline Anti-epilepsy activity in vivo & AEP & $\begin{array}{l}\text { Wang JH et al. }{ }^{[39]} \\
\text { Zhang H et al. }{ }^{[40]}\end{array}$ \\
\hline Anti-vascular disease in vivo and vitro & $\begin{array}{l}\text { Martentoxin } \\
\text { SVAP }\end{array}$ & $\begin{array}{l}\text { Gao SJ et al. }{ }^{[41]} \\
\text { Wang JX et al. }{ }^{[44]}\end{array}$ \\
\hline
\end{tabular}

Table 1: Main pharmacological properities of Buthus martensii Karsch

\section{Pharmacological activities of peptides}

\section{Antimicrobial activity}

Antimicrobial peptides (AMPs) are widely distributed in various species including plants, insects, humans, as well as single-celled organisms [8]. The structure of AMPs is usually amphiphilic topology [9,10]. Recently, researchers have reported and identified some venom peptides from the scorpion $\mathrm{BmK}$, which are found to show antimicrobial activity in vivo and in vitro. Some of these peptides present highly amphiphilic structure. Xian et al. [11] identified two amphiphilic venom peptides BmKb1 and BmKn2 from Buthus martensii Kasch. The functional tests showed that BmKn2 exhibited strong inhibitory activities against both Grampositive and Gram-negative bacteria while BmKb1 had weaker activity than BmKn2 in suppressing the growth of these bacteria [11]. Furthermore, BmKn2-7 (Figure 1), a derivative of BmKn2 designed by Cao et al. [12] showed higher antibacterial activity against clinical antibiotic-resistant strains and increased inhibitory activity against both Gram-positive and Gram-negative bacteria. Additionally, the hemolytic activity of BmKn2-7 was distinctly decreased compared with BmKn2. Animal studies showed that the topical use of BmK2n-7 could protect the skin of mice which was infected by Staphylococcus aureus

Gao et al. [13] isolated two compounds, 3 $\beta$-acetoxyl,2,14,22-trihydroxy, 9-hydroxymethyl,9 $\alpha, 5 \beta, 14 \beta$-card-20(22)enoli (1; Figure 1) and 1,2,3,4-tetrahydro-6-hydroxy1-5-pyrimidinecarbox-aldehyde (2; Figure 1), from 70\% methanol extracts of Chinese scorpion BmK by medium pressure liquid chromatography and RP-HPLC analysis technology. Both compounds exerted inhibitory activities against the Gram-positive bacteria Bacillus subtilis. And the minimum inhibitory concentration of the two compounds is $15 \mu \mathrm{g} /$ $\mathrm{mL}$.

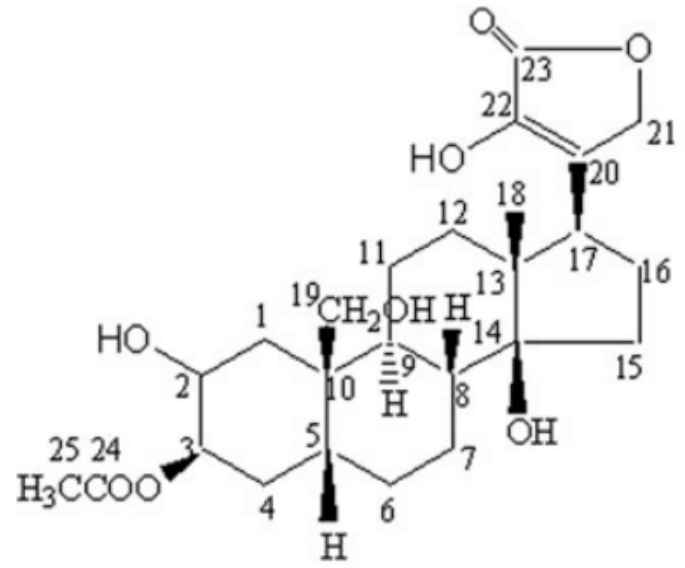

1

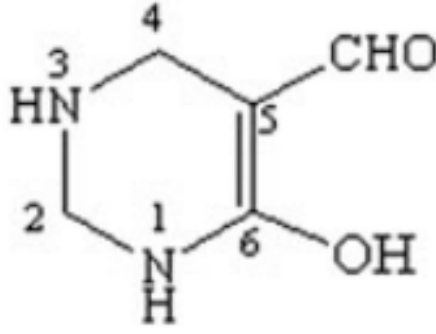

2

Figure 1: Structure of compound 1 and structure of compound 2

\section{Antitumor activity}

Gliomas cells can diffuse into the normal brain tissue. And Matrix metalloproteinase-2(MMP-2) and chloride ion channel play an important role in invasion of glioma cell [14,15]. Recently, researchers found that Cltx, as a specific chloride channel blocker, could bind specifically to glioma cell surface and inhibit the glioma cell migration. The first chlorotoxin (Cltx)-like peptide, BmK CT, isolated from the venom gland of BmK [16], is a novel blocker of the chloride ion channel and MMP-2 [17]. Yuejun Fu et al. [18] studied the influence of basic residues in BmK CT on the inhibitory activity to MMP-2 and proposed a model of BmK CT-MMP-2 catalytic domain complex to explain the relationship between the molecular structure of BmK CT and its mechanism in inhibition of glioma cell migration. Subsequently, Yuejun Fu et al. [19] found BmK CT expression was mediated by pEGFP-N1 and displayed a significant suppression in migration of rat glioma C6 cells by in vitro studies. Moreover, Yuejun Fu et al. [20] also performed a study to observe the combination of gene therapy pEGFP-N1-BmK CT with lithium chloride (LiCl) on C6 glioma cells. They reported that the combination therapy could inhibit the secretion and proliferation of pro-MMP2 in a synergistic manner, which might provide a new therapeutic strategy for glioma cancer. Besides, JianHua Shao et al. [21] isolated a dual-function peptide with analgesic and antitumor activities from scorpion venom. The peptide, BmK AGAP-SYPU2, showed antitumor effect in the Ehrlich ascites model and S-180 fibrosarcoma models in vivo. 


\section{Antinociceptive activity}

Whole scorpion, scorpion tails or their extracts are effective in soothing nerves and relieving pains caused by meningitis, cerebral palsy and rheumatism [22]. To date, more and more analgesic peptides from venom of the scorpion BmK have been found such as BmK IT2 [23], BmK dITAP3 [24], AngP1 [22], BmK AGP-SYPU1 [25-27], BmK AGP-SYPU2 [28-30], BmK AGAP [31-34] and BmKAS [35]. Moreover, Li et al. [36] reported that the venom of scorpion did not elicit dependence, while the analgesic substances such as morphine, heroin and aspirin have some side effects, in particular, the addictive nature of narcotic drugs. Therefore, the analgesic peptides from venom of scorpion $\mathrm{BmK}$ can be used as potential analgesic ingredients.

Depressant and excitatory insect neurotoxins: Scorpion neurotoxin can be divided into $\alpha$ - and $\beta$ - toxins. Scorpion $\alpha$ - toxins are further divided into three different pharmacological subfamilies including classical $\alpha$-mammal toxins, insect $\alpha$-toxins, $\alpha$-like toxins, which is according to their activities against mammals and insects [37]. In addition, neurotoxins also can be classified to depressant and excitatory toxins according to the symptomatology developed by injected animals [38]. BmK IT2 and BmK dITAP3 are depressant neurotoxins, which had been previously purified from venom of the scorpion BmK. The bioassay showed that both peptides could produce antinociception in rats and BmK dITAP3 exhibited 43\% inhibition efficiency for analgesia at the dosage of $5 \mathrm{mg} / \mathrm{kg}[23,24]$. AngP1, an excitatory insect neurotoxin, purified from scorpion BmK was reported to show 43\% inhibition efficiency for analgesia at a dose of $5 \mathrm{mg} / \mathrm{kg}$ on mice. And AngP1 was definitely devoid of mammalian toxicity [22].

BmK AGP-SYPU1: Yu Wang et al. [25] initially purified the analgesic peptides named BmK AGP-SYPU1 from the venom of Chinese scorpion BmK through a four-step chromatographic process. The recombinant BmK AGP-SYPU1 was also isolated from soluble fractions in the E. coli cell lysate and purified with metal chelating affinity column and cation exchange chromatography. The mouse twisting assay indicated that the native and recombinant BmK AGP-SYPU1 showed similar analgesic effects on mice. Subsequently, Yu Wang et al. [26] further investigated the relationship between the arginines in the C-terminal (65-67) of BmK AGP-SYPU1 and the analgesic activity. They found the arginines in the C-terminal were crucial for the analgesic activity and might be located at analgesic functional sites. Besides, the study by Li Deng et al. [27] identified a new relationship between the structure and analgesic activity of the scorpion BmK AGP-SYPU1. They performed a study to investigate the role of two conversed tyrosines (Tyr5 and Tyr42) from BmK AGP-SYPU1. The mouse-twisting test showed that Tyr5 and Tyr42 were involved in the analgesic activity. And the results of molecular simulation revealed that the core domain of BmK AGP-SYPU1 (Figure 2) was the key to analgesic activity and Tyr42 might be associated with pharmacological function when the core domain conformation was altered.

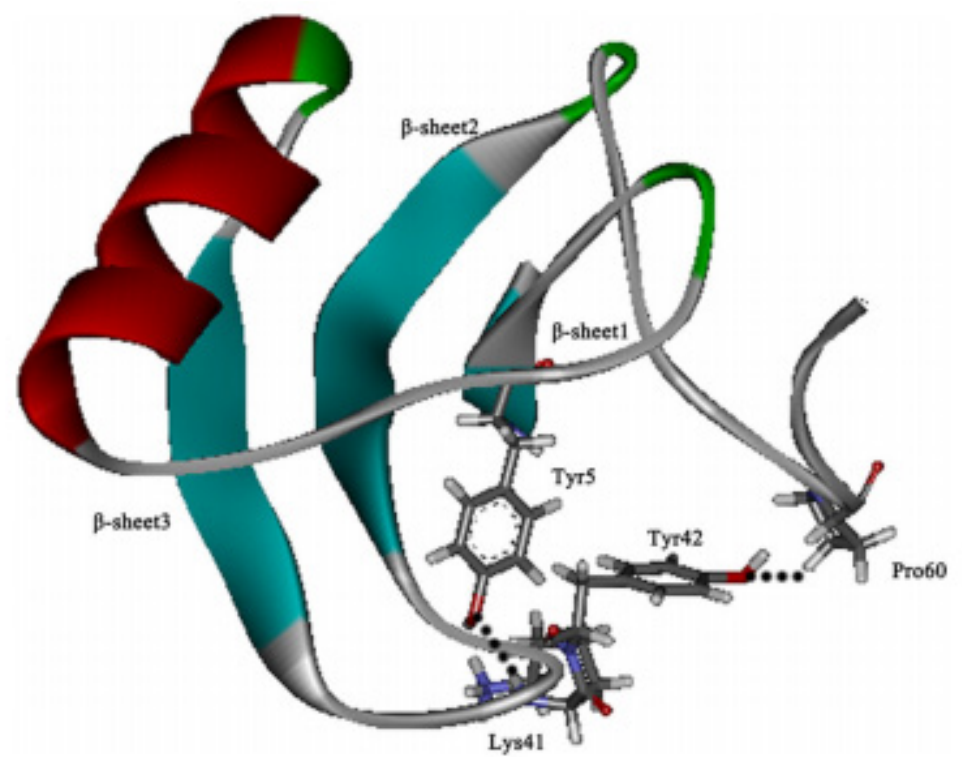

Figure 2: The final stable 3D structure of BmK AGP-SYPU1. The structural core of BmK AGP-SYPU1 is a conserved $\beta \alpha \beta \beta$ topology

The mouse writhing test by Rong Zhang et al. showed that BmK AGP-SYPU1 inhibited 70\% of the twisting action induced by acetic acid in mice at a dose of $0.1 \mathrm{mg} / \mathrm{kg}$ body weight [28].

BmK AGP-SYPU2: BmK AGP-SYPU2 is an analgesic peptide which is composed of 66 amino acid residues [28]. Rong Zhang et al. [28] purified BmK AGP-SYPU2 from the Chinese scorpion BmK through five continuous chromatographic steps. They identified the target peptides in every separation step by the mouse twisting model test and determined the molecular weight, isoelectric point and N-terminal sequence of the peptide. The mouse writhing test showed that BmK AGP-SYPU2 inhibited 64.3\% of the twisting action induced by acetic acid in mice at a dose of $0.1 \mathrm{mg} / \mathrm{kg}$ body weight [28]. The functional role of glycines in the C-terminal end of BmK AGP-SYPU2 was investigated by Rong Zhang et al. [29]. They detected the analgesic activity of nature BmK AGP-SYPU2 and its mutants which were obtained by site-directed mutagenesis. When the glycines at the C-terminal end 
altered, the analgesic activity of mutant was significantly decreased. The study indicated that glycines at the C-terminal end played an important role for the analgesic activity of BmK AGP-SYPU2. Subsequently, Yongshan Zhao et al. [30] further investigated the relationship between the C-terminal segment of BmK AGP-SYPU2 and their analgesic activity by performing three molecular dynamics simulations (BmK AGP-SYPU2 structure and its two mutants of its structure). The data of these calculations suggested that the glycine residues at the C-terminal segment stabilized the protruding topology of the NC domain, which was an important decisive factor for the analgesic activity of BmK AGP-SYPU2.

AGAP: Antitumor-analgesic peptide (AGAP) has both analgesic and antitumor activity which was purified from the venom of the Chinese scorpion BmK. Yanfeng Liu et al. [31] reported the expression and purification of AGAP in Escherichia coli and demonstrated the analgesic and antitumor activities of AGAP on mice. Rui Ma et al. [32] looked for the analgesic domains by using the conformational stability, catalytic activity and folding, and site-directed mutagenesis in disulfide bridges and examined the analgesic activity of 12 mutants through the mouse-twisting assay. They found the key to the analgesic activity, which was called "core domain". In addition, Yong Cui et al. [33] studied the role of residues in AGAP by using an effective Escherichia coli expression system and site-directed mutagenesis. They evaluated the extent to which residues of AGAP contributed to its analgesic activity and pinpointed the important residue for the analgesic activity by replacing all of these residues individually with one amino acid.

Qinghong Mao et al. [34] investigated the antinociceptive effect and underlying mechanism of AGAP from scorpion BmK. AGAP were injected in doses of 0.2, 1 and $5 \mathrm{mg}$ intraplantarly (i.pl.) at $10 \mathrm{~min}$ before formalin injection in mice. The number of flinching paw showed that formalin-induced inflammatory pain could be suppressed by pre-intraplantar injection of AGAP in a dose-dependent manner through a MAPKs-mediated mechanism in mice. Furthermore, AGAP could enhance the effects of the inhibitors of MAPKs on the inflammatory pain.

\section{Anti-epilepsy activity}

In China, scorpions have been used to treat epilepsy and convulsion by Chinese traditional doctors since the Sung Dynasty (A.D. 960-1279) [39].

The first anti-epilepsy peptide (AEP) was isolated and purified from venom of scorpion BmK by Zhou et al. [1]. The peptide is composed of 66 residues from 18 amino acids and has molecular weight of 8290. The pharmacological test suggested that AEP could suppress epilepsy induced by coriaria lactone and cephaloridine and had no side effect on heart rate and blood pressure in rats even after the dose reached $28 \mathrm{mg} / \mathrm{kg}$ body weight. Chunguang Wang et al. [40] further confirmed its anti-epilepsy effect on rat. They found BmK AEP showed anti-epilepsy activity when at the dose of $0.057 \mu \mathrm{gg}^{-1}$ body weight. Chunguang Wang et al. [40] also found the toxicity of BmK AEP in mammalian and insects was very weak even the dosage of BmK AEP was up to $20 \mu \mathrm{g}$ and $2 \mu \mathrm{g}$, respectively. While the typical mammalian toxins, such as BmK M1 and the excitatory insect toxin BmK IT-AP could cause paralysis. The cDNA sequence of BmK AEP showed that it shared homology with other depressant insect toxins, but there was a remarkable difference between them mainly focused at residues 6, 7 and 39, which might relate to the unique action of BmK AEP.

\section{Anti-vascular disease}

In certain vascular disease, nitric oxide (NO) can be released from the endothelium, which is one of the most important vasoactive substances [41]. NO is produced by nitric oxide synthase (NOS) which includes neuronal NOS (nNOS), endothelial NOS (eNOS) and inducible NOS (iNOS) in mammals $[42,43]$.

$\mathrm{NO}$ release in endothelium is associated with Hyperpolarization which is caused by the activation of $\mathrm{Ca}^{2+}$-activated $\mathrm{K}^{+}$channels (BKCa channels) Jun Wang et al. [41] purified a large-conductance $\mathrm{Ca}^{2+}$-activated $\mathrm{K}^{+}$channel inhibitor, martentoxin, from $\mathrm{BmK}$ and demonstrated its protective effects in inflammatory vascular diseases. They found martentoxin decreased NO production and iNOS activation induced by TNF- $\alpha$. They also demonstrated that martentoxin retarded the down-regulation of eNOS mRNA induced by TNF- $\alpha$ in human umbilical vein endothelial cells (HUVECs).

Yimin Song et al. [44] performed studies to investigate effects of scorpion venom active polypeptide (SVAP) from scorpion BmK on platelet aggregation in ex vivo and vitro in rabbits, thrombosis in carotid artery of rats as well as plasma 6-keto-PG $\mathrm{F}_{1 \alpha}$ and TXB in rats. The results showed that SVAP significantly inhibited the rabbit platelet aggregation triggered by thrombin and ADP in vitro and at the dose of $0.32,0.64 \mathrm{mg} / \mathrm{kg}$ iv extended the occlusion time of thrombosis induced by electrical stimulation. Additionally, SVAP could distinctly increase the plasma concentration of 6-keto-PG $\mathrm{F}_{1 \alpha}$ and value of $\mathrm{PG} \mathrm{I}_{2} / \mathrm{TXA}_{2}$, but slightly affect rats plasma concentration of $\mathrm{TXB}_{2}$ in vitro and in ex vivo, which indicated the mechanism of the antithrombotic action of SVAP.

\section{Other beneficial effects}

There are not only these pharmacological effects of BmK described above, but also other beneficial effects. As is well known, scorpion and its venom have been used in Traditional Chinese Medicine to treat chronic neurological disorders. The mechanism about that was studied. Tao Wang et al. [45] purified scorpion venom heat-resistant peptide (SVHRP) from scorpion BmK, confirmed its neurotrophic effect in vitro and in vivo and explored associated molecular mechanisms. They found the concentrations of BDNF, NGF and GDNF were higher in the conditioned medium of SVHRP-pretreated astrocytes, in contrast to normal astrocyteconditioned medium. And more GFAP-positive cells were detected in SVHRP treated mouse brain. Furthermore, BmKbpp, a 47 
amino acid peptide, was demonstrated that it acted as a signaling molecule involving innate immune regulation at low concentrations and displayed bradykinin potentiating and immune-modulatory activities [46]. As described above, the peptides SVAP purified from scorpion BmK have effects of inhibiting the rabbit platelet aggregation and thrombosis. One study indicated that SVAP also could improve mesenteric microcirculation [47].

\section{Conclusion}

Scorpion Buthus martensii Karsch are rich in peptides which represent a tremendous unexplored resource for use in drug design and development. For now, more and more researchers study the role of amino acids in pharmacological activities and characterize the structure-function relationships of bioactive peptides in order to design the peptides with higher bioactivity by using sitedirected mutagenesis and gene cloning technology. Based on the identified peptides purified from scorpion Buthus martensii Karsch, some researchers have tried to establish a new, economical and effective system for industrial peptides production with the use of molecular cloning technique [48]. However, some bioactivity peptides have not been identified and the mechanism of some pharmacological effects is not clarified. Furthermore, though the scorpion has been traditionally used as a source of drugs, the clinical studies about single peptide are few. Careful clinical studies comparing pharmacological activities of the whole scorpion, scorpion venom, the extracts of them and the single peptides still need to determine whether the single peptides of scorpion Buthus martensii Karsch provide real clinical benefits.

\section{References}

1. Zhou XH, Yang D, Zhang JH, Liu CM, Lei KJ (1989) Purification and Nterminal partial sequence of anti-epilepsy peptide from venom of the scorpion Buthus martensii Karsch. Biochem J 257: 509-17.

2. State Pharmacopoeia Committee of the People's Republic of China 2000.

3. Catterall WA (1980) Neurotoxins that act on voltage sensitive sodium channels in excitable membranes. Annu Rev Pharmacol Toxicol 20: 15-43.

4. Carbone E, Wanke E, Prestipino G, Possani LD, Maelicke A (1982) Selective blockage of voltage-dependent K+-channels by a novel scorpion toxin. Nature 296: 90-1.

5. Miller C, Moczydlowski E, Latorre R, Phillips M (1985) Charybdotoxin, a protein inhibitor of single Ca2+-activated K+ channels from mammalian skeletal muscle. Nature 313: 316-8.

6. Debubm JA, Maggio JE, Strichartz GR (1993) Purification and characterization of chlorotoxin, a chloride channel ligand from the venom of the scorpion. Am J Physiol 264: C361-9.

7. Valdivia HH, Possani LD (1998) Peptide toxins as probes of ryanodine receptor structure and function. Trends Cardiovasc Med 8: 111-8.

8. Maróti G, Kereszt A, Kondorosi E, Mergaert P (2011) Natural roles of antimicrobial peptides in microbes, plants and animals. Res Microbiol 162: 363-74.

9. Godballe T, Nilsson LL, Petersen PD, et al. (2011) Antimicrobial betapeptides and alpha-peptoids. Chem Biol Drug Des 77: 107-16.

10. Zasloff M (2002) Antimicrobial peptides of multicellular organisms. Nature 415: 389-95.

11. Zeng XC, Wang SX, Zhu Y, Zhu SY, Li WX (2004) Identification and functional characterization of novel scorpion venom peptides with no disulfide bridge from Buthus martensii Karsch. Peptides 25: 143-50.

12. Cao L, Dai C, Li Z, Fan Z, Song Y, et al. (2012) Antibacterial activity and mechanism of a scorpion venom peptide derivative in vitro and in vivo. Plos One 7: 40135-44.

13. Gao J, Yin W, Gao T, Deng R, Li X (2014) Two bioactive compounds from the Chinese scorpion Buthus martensii Karsch. Nat Prod Res 28: 698-703.

14. Deshane J, Garner CC, Sontheimer H (2003) Chlorotoxin inhibits glioma cell invasion via matrix metalloproteinase-2. J Biol Chem 278: 4135-44.

15. Olsen ML, Schade S, Lyons SA, Amaral MD, Sontheimer H (2003) Expression of voltage-gated chloride channels in human glioma cells. J Neurosci 23: $5572-82$.

16. Zeng XC, Li WX, Zhu SY, Peng F, Zhu ZH, et al. (2000) Cloning and characterization of a cDNA sequence encoding the precursor of a chlorotoxin-like peptide from the Chinese scorpion Buthus martensii Karsch. Toxicon 38: 1009-14.

17. Fu YJ, Yin LT, Liang AH, Zhang CF, Wang W, et al. (2007) Therapeutic potential of chlorotoxin-like neurotoxin from the Chinese scorpion for human gliomas. Neurosci Lett 412: 62-7.

18. Fu YJ, An N, Chan KG, Wu YB, Zheng SH, et al. (2011) A model of BmK CT in inhibiting glioma cell migration via matrix metalloproteinase-2 from experimental and molecular dynamics simulation study. Biotechnol Lett 33: 1309-17.

19. Fu Y, Jiao Y, An N, Liang A (2012) pEGFP-N1-mediated BmK CT expression suppresses the migration of glioma. Cytotechnology 65: 533-9.

20. Fu YJ, Jiao YM, Zheng S, Liang A, Hu F (2014) Combination of lithium chloride and pEGFP-N1-BmKCT effectively decreases proliferation and migration of C6 glioma cells. Cytotechnology doi 10.1007/s10616-014-9768-2.

21. Shao JH, Cui Y, Zhao MY, Wu CF, Liu YF, et al. (2013) Purification, characterization, and bioactivity of a new analgesic-antitumor peptide from Chinese scorpion Buthus martensii Karsch. Peptides 53: 89-96.

22. Guan RJ, Wang M, Wang D, Wang DC (2001) A new insect neurotoxin AngP1 with analgesic effect from the scorpionButhus martensii Karsch: purification and characterization. J Pept Res 58: 27-35.

23. Wang CY, Tan ZY, Chen B, Zhao ZQ, Ji YH (2000) Antihyperalgesia effect of BmK IT2, a depressant insect-selective scorpion toxin in rat by peripheral administration. Brain Res Bull 53: 335-8.

24. Guan R, Wang CG, Wang M, Wang DC (2001) A depressant insect toxin with a novel analgesic effect from scorpion Buthus martensiiKarsch. Biochim Biophys Acta 1549: 9-18.

25. Wang Y, Wang L, Cui Y, Song YB, Liu YF, et al. (2011) Purification, characterization and functional expression of a new peptide with an analgesic effect from Chinese scorpion Buthus martensii Karsch (BmK AGP-SYPU1). Biomed Chromatogr 25: 801-7.

26. Wang Y, Song YB, Yang GZ, Cui Y, Zhao YS, et al. (2012) Arginine residues in the C-terminal and their relationship with the analgesic activity of the toxin from the Chinese scorpion Buthus martensii Karsch (BmK AGP-SYPU1). Appl Biochem Biotechnol 168: 247-55. 
27. Deng L, Zhang HX, Wang Y, Zhang R, Wen X, et al. (2014) Site-directed mutagenesis of BmK AGP-SYPU1: the role of two conserved Tyr (Tyr5 and Tyr42) in analgesic activity. Protein J 33: 157-64.

28. Zhang R, Yang Z, Liu YF, Cui Y, Zhang JH (2011) Purification, characterization and cDNA cloning of an analgesic peptide from the Chinese scorpion Buthus martensii Karsch (BmK AGP-SYPU2). Mol Biol 45: 956-62.

29. Zhang R, Cui Y, Zhang X, Yang Z, Zhao Y, et al. (2010) Soluble expression, purification and the role of C-terminal glycine residues in scorpion toxin BmK AGPSYPU2. BMB Rep 43: 801-6.

30. Zhao YS, Zhang R, Xu Y, Cui Y, Liu YF, et al. (2013) The role of glycine residues at the C-terminal peptide segment in antinociceptive activity: a molecular dynamics simulation. J Mol Model 19: 1295-9.

31. Liu YF, Ma RL, Wang SL, Duan ZY, Zhang JH, et al. (2003) Expression of an antitumor-analgesic peptide from the venom of Chinese scorpion Buthus martensii karsch in Escherichia coli. Protein Expr Purif 27: 253-8.

32. Ma R, Cui Y, Zhou Y, Bao YM, Yang WY, et al. (2010) Location of the analgesic domain in Scorpion toxin BmK AGAP by mutagenesis of disulfide bridges. Biochem Biophys Res Commun 394: 330-4.

33. Cui Y, Guo GL, Ma L, Hu N, Song YB, et al. (2010) Structure and function relationship of toxin from Chinese scorpion Buthus martensii Karsch (BmKAGAP): gaining insight into related sites of analgesic activity. Peptides 31: 995-1000.

34. Mao Q, Ruan J, Cai X, Lu W, Ye J, et al. (2013) Antinociceptive Effects of Analgesic-Antitumor Peptide (AGAP), a Neurotoxin from the Scorpion Buthus martensiiKarsch, on Formalin-Induced Inflammatory Pain through a Mitogen-Activated Protein Kinases-Dependent Mechanism in Mice. Plos One 8: e78239.

35. Cui Y, Song YB, Ma L, Liu YF, Li GD, et al. (2010) Site-directed mutagenesis of the toxin from the Chinese scorpion Buthus martensii Karsch (BmKAS): insight into sites related to analgesic activity. Arch Pharm Res 33: 1633-9.

36. Li F, Lu SN, Pan LS (1997) The experimental evaluation of scorpion toxins physical dependence. J. Pharmacol. Toxin. 11: 154-8.

37. Goudet C, Chi CW, Tytgat J (2002) An overview of toxins scorpion and genes from the venom of the Asian Buthus martensi Karsch. Toxicon 40: 1239-58.

38. Zlotkin E (1991) Venom neurotoxins - models for selective insecticides. Phytoparasitica 19: 177-82.

39. Zhou XH (1984) Shengwu Huaxue Yu Shengwu Wuli Jinzhan 56: 20-9.

40. Wang CG, He XL, Shao F, Liu W, Ling MH, et al. (2001) Molecular characterization of an anti-epilepsy peptide from the scorpion Buthus martensi Karsch. Eur J Biochem 268: 2480-5.

41. Wang J, Qian W, Zhu Q, Chen J, Huan F, et al. (2013) Martentoxin, a large-conductance Ca2+-activated K+ channel inhibitor, attenuated TNF- $a$-induced nitric oxide release by human umbilical vein endothelial cells. J Biomed Res 27: 386-93.

42. Stuehr DJ (1999) Mammalian nitric oxide synthases. Biochim Biophys Acta 1411: 217-30.

43. Stuehr DJ, Santolini J, Wang ZQ, Wei CC, Adak S (2004) Update on mechanism and catalytic regulation in the NO synthases. J Biol Chem 279: $36167-70$.

44. Song YM, Tang XX, Chen XG, Gao BB, Gao Er, et al. (2005) Effects of scorpion venom bioactive polypolypeptides on platelet aggregation and thrombosis and plasma 6-keto-PG F1 $\alpha$ and TXB2 in rabbits and rats. Toxicon 46: 230-5.

45. Wang T, Wang SW, Zhang Y, Wu XF, Peng Y, et al. (2014) Scorpion Venom Heat-Resistant Peptide (SVHRP)Enhances Neurogenesis and Neurite Outgrowth of Immature Neurons in Adult Mice by Up-Regulating BrainDerived Neurotrophic Factor (BDNF). Plos One 9: $9977-86$.

46. Zeng XC, Wang S, Nie Y, Zhang L, Luo X (2012) Characterization of BmKbpp, a multifunctional peptide from the Chinese scorpion Mesobuthus martensii Karsch: gaining insight into a new mechanism for the functional diversification of scorpion venom peptides. Peptides 33: 44-51.

47. Song YM, Li XK, Zou L, Gao E, Lv XR, et al. (2002a) Effects of scorpion venom active polypeptides on mesenteric microcirculation of rats. Chin J Microcircul 12:15-6.

48. Song YB, Huang TT, Lai LL, Zhou J, Yang WY, et al. (2011) Expression of Anti-Neuroexcitation Peptide III of Scorpion Buthus martensiiKarsch BmK ANEP III in Plants. Molecular Biology 45: 949-55.

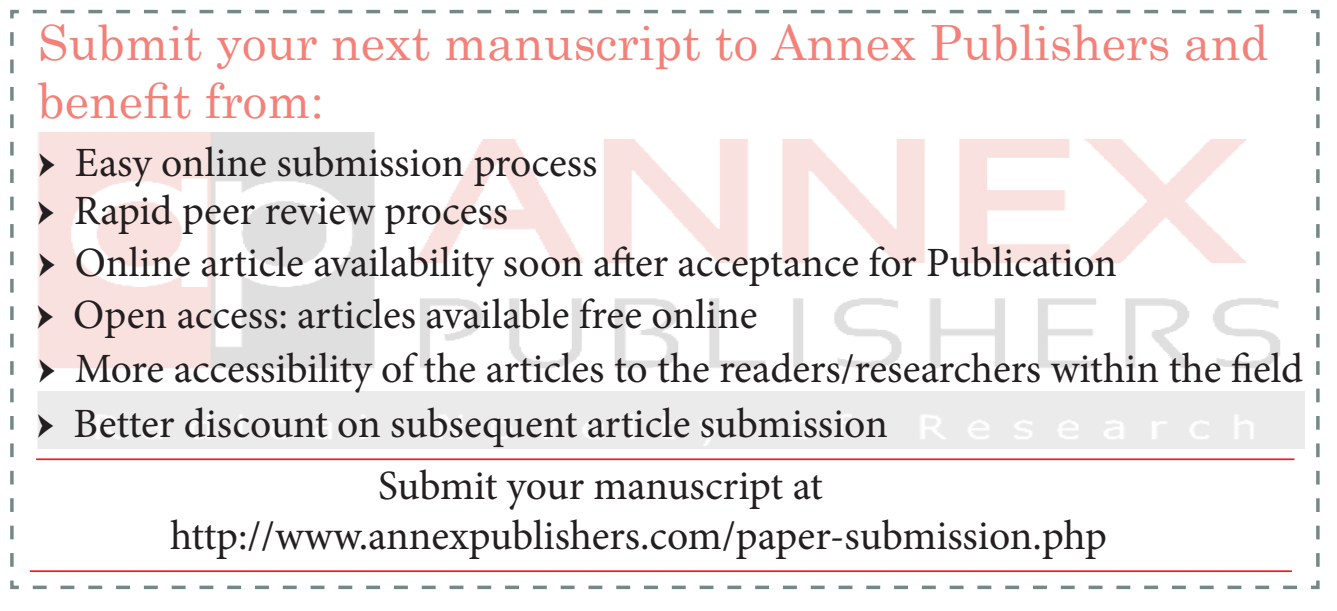

